\title{
Fixed-dose combination therapy for Parkinson's disease with a spotlight on entacapone in the past 20 years: a reduced pill burden and a simplified dosing regime
}

\author{
András Salamon, Dénes Zádori , László Szpisjak , Péter Klivényi \& László \\ Vécsei
}

To cite this article: András Salamon, Dénes Zádori , László Szpisjak, Péter Klivényi \& László Vécsei (2020): Fixed-dose combination therapy for Parkinson's disease with a spotlight on entacapone in the past 20 years: a reduced pill burden and a simplified dosing regime, Expert Opinion on Pharmacotherapy, DOI: 10.1080/14656566.2020.1806237

To link to this article: https://doi.org/10.1080/14656566.2020.1806237

\section{曲 Published online: 18 Aug 2020.}

\section{Submit your article to this journal $₫$}

Q View related articles $₫$

View Crossmark data $\nearrow$ 


\title{
Fixed-dose combination therapy for Parkinson's disease with a spotlight on entacapone in the past 20 years: a reduced pill burden and a simplified dosing regime
}

\author{
András Salamon ${ }^{a}$, Dénes Zádoria, László Szpisjaka , Péter Klivényi ${ }^{a}$ and László Vécseia,b \\ ${ }^{a}$ Department of Neurology, Faculty of Medicine, Albert Szent-Györgyi Clinical Center, University of Szeged, Szeged, Hungary; ${ }^{b}$ Department of \\ Neurology and Interdisciplinary Excellence Centre, Faculty of Medicine, MTA-SZTE Neuroscience Research Group, Szeged, Hungary
}

\section{ABSTRACT}

Introduction: Parkinson's disease (PD) is a progressive, chronic neurodegenerative disorder. The main neuropathological cause of the disease is the death of dopaminergic neurons in the substantia nigra. Unfortunately, there is no curative treatment yet. The gold-standard of the treatment is levodopa (LD). During the course of the disease, motor complications develop, which postulates the addition of entacapone (ENT) to the dopaminergic medication. Previous studies have suggested that patients have a better quality of life when entacapone is added in a combination with LD.

Areas covered: A systematic literature search was performed. Articles were identified through PubMed (MEDLINE), Web of Science, Ovid, and ClinicalTrials.gov databases. The following search terms were used: 'Levodopa' AND 'Carbidopa' OR 'Benserazide' AND 'Entacapone'. The search period was between 2000 and 2020. Twenty randomized and 10 non-randomized clinical trials (12,893 subjects) were included in the qualitative analysis. The systematic review was written in line with the PRISMA guideline.

Expert opinion: ENT administered in combination with LD resulted in a better quality of life compared to separate tablets. Therefore, in PD patients where impaired motor performance develops and the application of entacapone is necessary, it is suggested to be administered in a single tablet form.
ARTICLE HISTORY

Received 14 February 2020 Accepted 3 August 2020

\section{KEYWORDS}

Adherence; combination; entacapone; fixed-dose; non-motor symptoms; Parkinson's disease; UPDRS

\section{Introduction}

Parkinson's disease is the second most common neurodegenerative disorder [1]. The estimated prevalence is around 10-18 per 100000 [2]. The most important motor symptoms are bradykinesia, tremor, and/or rigidity [2]. The main neuropathological cause of the disease is the death of the dopaminergic neurons in the substantia nigra [3]. Unfortunately, there is no curative treatment yet, however extensive preclinical and clinical studies are ongoing [4]. Today, the focus of treatment is on the compensation of the hypodopaminergic state of the brain with exogenous levodopa (LD) substitution [5]. In general, a significant proportion of levodopa is rapidly metabolized by the peripheral aromatic amino-acid decarboxylase (AADC) [6-8]. To prevent this process, dopa-decarboxylase inhibitors $(\mathrm{DDCl})$ have been introduced in daily clinical practice (benserazide (B) and carbidopa (CD)) [6-8]. However, $A A D C$ is not the only enzyme which is involved in this metabolic pathway [6-8]. Catechol-O-methyltransferase (COMT) can also convert LD to 3-O-methyldopa (3-OMD) [6-8]. To block this pathway as well, three widely known COMT inhibitors (entacapone (ENT), tolcapone (TLC), and opicapone (OPC)) have been introduced [6-8]. Although ENT is the most widely used COMT inhibitor, it requires multiple daily doses. In contrast, it is sufficient to administer OPC once a day. The only central acting COMT inhibitor is TLC; however, due to its hepatotoxic effects, it should be closely monitored [6]. The treatment of patients with Parkinson's disease becomes very complicated as the disease progresses [5]. The 'ON'-time will get shorter and the number of hours with inappropriate movement increases [5]. Fractionation and intensification of the LD treatment gradually become necessary [5]. If end-ofdose motor fluctuations develop, an option is to introduce the COMT-inhibitors in combination with LD/DDCI [5]. In addition to the motor symptoms of the disease, many non-motor symptoms, including Parkinson's dementia are known [9]. Given that the treatment strategy gets more complicated as the disease progresses and, simultaneously, the condition of the patient gradually deteriorates, combination therapies play a major role in achieving optimal compliance and therapeutic response [5]. Furthermore, the cost-effectiveness of combination therapies is not negligible [10].

For the reasons mentioned above, the primary aim of this systematic review is to summarize the efficacy data on entacapone as an adjunct therapy to LD on motor fluctuations (in line with PRISMA criteria [11]). Furthermore, the secondary objective is to compare the pharmacological and quality of life effects of two modes of oral ENT administration (LD/DDCI plus ENT separately versus $\mathrm{LD} / \mathrm{DDCl} / \mathrm{ENT}$ ). An additional purpose of our study is to demonstrate the importance of 


\section{Article highlights}

- Parkinson's disease is the second most common neurodegenerative disorder with an estimated prevalence of around 10-18 persons per 100000.

- The gold-standard of the treatment is levodopa (LD). However, during the course of the disease, motor complications develop which often leads the prescription of entacapone (ENT) in addition to the dopaminergic medication.

- ENT is a peripherally acting COMT-inhibitor.

- ENT administered in combination (LD/CD/ENT) results in a better quality of life compared to the drugs administered separately (LD/ $\mathrm{CD}+\mathrm{ENT}$ or LD/B + ENT)

- The cost-effectiveness of combination formulations may be an important future aspect for the patient's and health insurance's budget, especially as an increased QoL could increase a patient's number of active years and reduce the need for hospital care.

This box summarizes key points contained in the article.

combination therapies in Parkinson's disease, using the example of LD/CD/ENT.

\section{Methods}

\subsection{Eligibility criteria}

English language, available online reviews, editorial articles, and original publications have been included in the systematic literature analysis, as well as clinical trials with accessible results. The search period was between 2000 and 2020 (January). Clinical trials on individuals below the age of 18 are not included in the analysis. The main focus of the literature search was on the effect of ENT on the motor performance of Parkinsonian patients. Due to the lack of LD combination formulation for OPC and TLC, it was not possible to compare these with ENT combinations. For this reason, OPC and TLC were beyond the scope of this article. Furthermore, the studies addressing the impact of ENT on levodopa-induced hyperhomocysteinemia and vitamin B12 deficiency were also excluded.

\subsection{Information sources and search strategy}

Articles were identified through PubMed (MEDLINE), Web of Science, Ovid, and ClinicalTrials.gov databases. The following search terms were used in all applied online databases: 'Levodopa' AND ‘Carbidopa' OR 'Benserazide' AND ‘Entacapone'.

\subsection{Data items}

The following information was searched in the identified publications: (1) type of the trial; (2) participant characteristics (number of included subjects, inclusion, and exclusion criteria); (3) purpose of the study; (4) intervention and groups; (5) duration of the study; (6) clinical assessment scales; (7) outcome measures (primary and secondary); (8) main findings.

\section{Results}

\subsection{Study selection process}

Through PubMed (MEDLINE) searches 179 items were identified (Figure 1). Using the Web of Science, Ovid, and ClinicalTrials.gov databases, we identified an additional 497 items. Duplications were removed using Mendeley software $(n=208)$. After screening the 468 identified findings (titles and abstracts were read), 39 articles were eligible for full-text review. After the detailed evaluation of the above-mentioned texts, 30 studies were included in this systematic analysis.

\subsection{Study characteristics}

Study type - 20 randomized (double-blind, cross-over $(\mathrm{n}=8$ ); double-blind, parallel-group ( $\mathrm{n}=5)$; single-blind, cross-over ( $n=1)$; single-blind, parallel-group $(n=1)$; open-label, crossover $(n=2)$; open-label, parallel-group $(n=3)$ ) and 10 nonrandomized (open-label) clinical trials were identified (Table 1).

Number and characteristics of participants - 12,893 subjects (male and female patients) were involved in these studies (PD patients $=12,784$; healthy subjects $=109$ ). Age range: 30 to 80 years.

Inclusion and exclusion criteria (PD population) - The most widely used inclusion criteria were the following: (1) - idiopathic PD; (2) - Hoehn-Yahr stage 1 to 3; (3) - motor fluctuation information (absent; no or minimal, nondisabling motor fluctuation; 'end-of-dose type'; wearing-off; on-off phenomenon; early end-of-dose wearing-off defined by QUICK questionnaire; mild wearing-off phenomena; without unpredictable fluctuations; wearing-off with or without mild dyskinesia; at least 1 'yes' on the Motor Fluctuation Questionnaire) (4) - LD dose (stable dose; not optimally treated) and formulation information (standard; IR; SR; RR). The main exclusion criteria used in the studies were the following: (1) - secondary or atypical parkinsonism; (2) - severe systemic or psychiatric illness; (3) - previous or current treatment which interferes with the tested drug; (4) - previous treatment with ENT; (5) - motor performance information (unpredictable dyskinesia; unpredictable "OFF" periods; painful dyskinesia; disabling dyskinesia; unpredictable fluctuations; complex motor fluctuations; severe dyskinesia; more than mild dyskinesia).

Duration of the study - the duration of the studies ranged from 2 days to 136 weeks.

Clinical assessment scales - the following tests were generally used in the identified clinical trials: (1) - UPDRS scale; (2) - PDQ-39 and -8; (3) - SF-36; (4) - PSI; (5) - VAS; (6) - Clinical Global Impression (patient and investigator); (7) - Motor fluctuations Questionnaire; (8) - QoL; (9) - ESS; (10) - MMSE; (11) - Schwab and England ADL scores; (12) - BDI; (13) - Wearing Off Card; (14) Motor performance tests (grip strength, line tracing test, peg insertion test); (15) - pharmacokinetic test; (16) - LD dose equivalent.

\subsection{Main findings}

\subsubsection{Pharmacokinetic data}

The majority of the performed pharmacokinetic studies focused on the effect of ENT on LD in different administration 


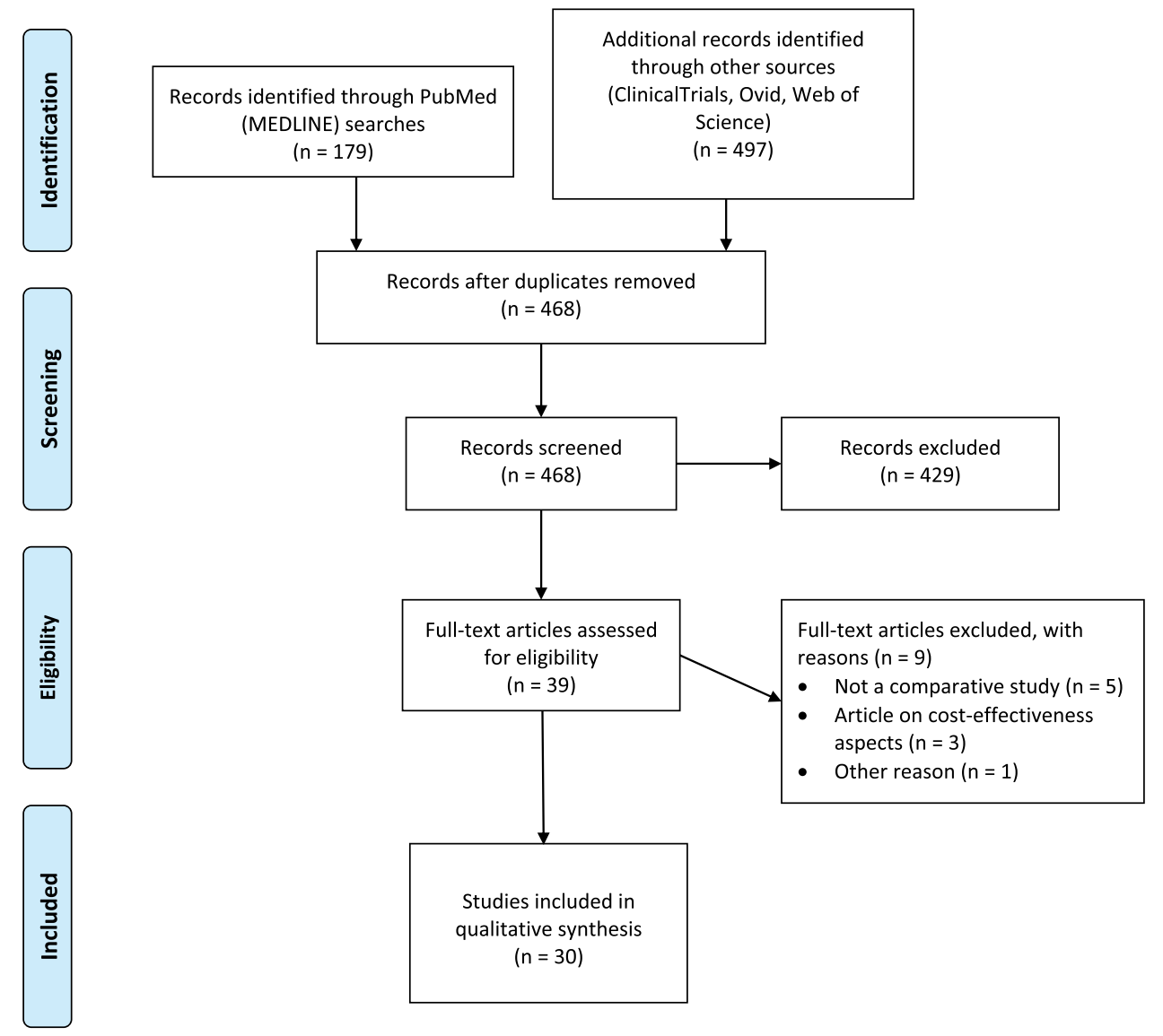

Figure 1. PRISMA flow diagram.

and combination settings. There was no relevant pharmacokinetic difference between LD/CD and LD/B after ENT administration. However, other authors [16] suggested, that LD/B may have more significant AADC inhibitory effects. Addition of ENT to $L D / C D$ or LD/B resulted in increased AUC and decreased 3-OMD levels (regardless of the type of the LD formulation (e.g. RR, CR, IR; separately administered or single tablet form)) [17-24].

\subsubsection{Scale-based assessments, motor performance}

To estimate the alteration in motor performance, UPDRS scale was the most widely used [26-37 and NCT00391898, NCT00642356]. The overall conclusion of many of the identified studies was that regardless of the administration form of ENT (administered separately or in single tablet form), motor performance improved [20,25-27]. This positive effect is also detectable in different subpopulations (e.g. in early-stage PD patients) [25]. However, in the STRIDE-PD clinical trial, an earlier appearance and increased frequency of dyskinesias were detected in the ENT group [26]. Interestingly, in the SIMCOM study, a pronounced improvement was found in the UPDRS score (the mean UPDRS score (parts III) improved significantly (from 24.0 by $1.9 ; p<0.01$ )) with the single tablet LD/CD/ENT group compared to LD/CD plus ENT (separately) group [28]. The repeated administration of ENT containing LD combination resulted in significantly better motor scores (UPDRS) and performance in comparison to repeated administration of LD/ $C D$ alone, meanwhile, there was lesser pronounced fluctuation of movements [23]. In the START-M trial - similarly to the TCINIT study - a switch from the previous LD medication to LD/ CD/ENT resulted in a $29 \%$ reduction rate on the UPDRS [29].

\subsubsection{Quality of life}

The effect of entacapone addition to $L D / D D C l$ and the formulation-related effects (LD/DDCI + ENT vs. LD/CD/ENT) were also examined from the perspective of QoL.

Hauser et al. found over 39 weeks, in early PD populations, that $\mathrm{LD} / \mathrm{CD} / \mathrm{ENT}$ resulted in greater clinical improvement than LD/CD alone [25]. The risk of motor complications was not elevated in the ENT group [25]. ADL (Part II, UPDRS, $p=0.025$ ) and Schwab and England scores (by patient: $p=0.006$, by rater: 0.003$)$ were significantly better in the LD/CD/ENT group [25]. There was a similar tendency with the PDQ-39 and PDQ-8 scores [25]. The p-CGI was significantly better as well in the above-mentioned group (LD/CD group - 34.8\% reported that they were 'much improved'; however, in the LD/CD/ENT group it was 36.7\%) [25]. Another study found that in patients without motor complications, separately administering ENT for 21 weeks did not improve the ADL section of UPDRS scale [30]. However, this treatment resulted in a significant improvement in the QoL measures (PDQ-39 ( $p=<0.01$ ), SF-36 (vitality domain $-p=0.04$; physical component $-p=0.009)$, PSI 

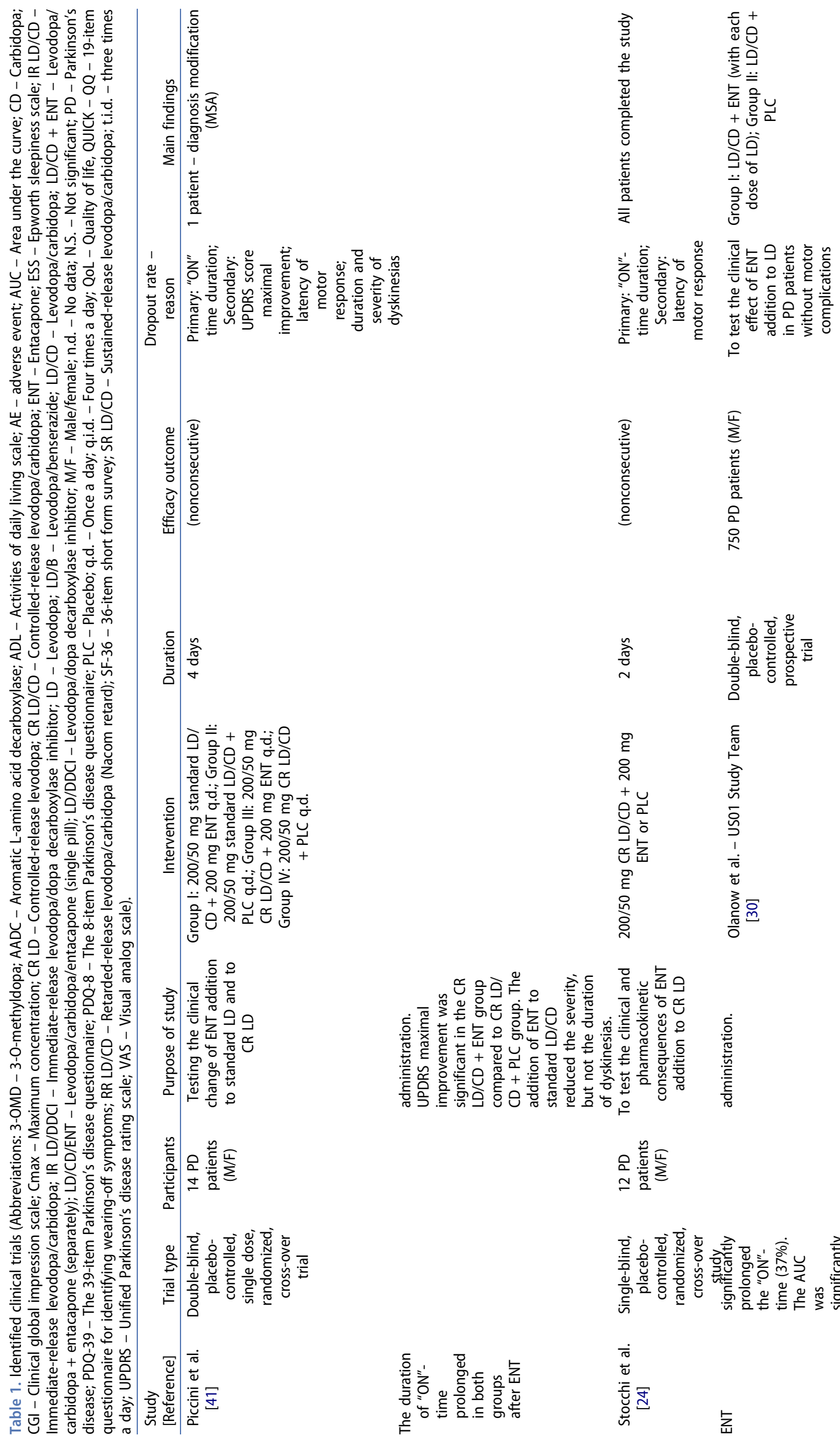

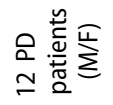

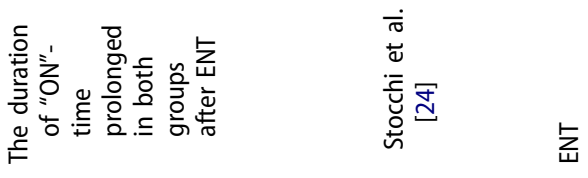

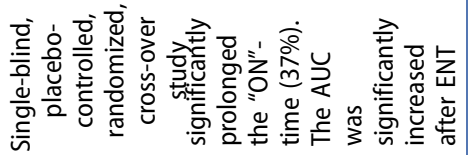




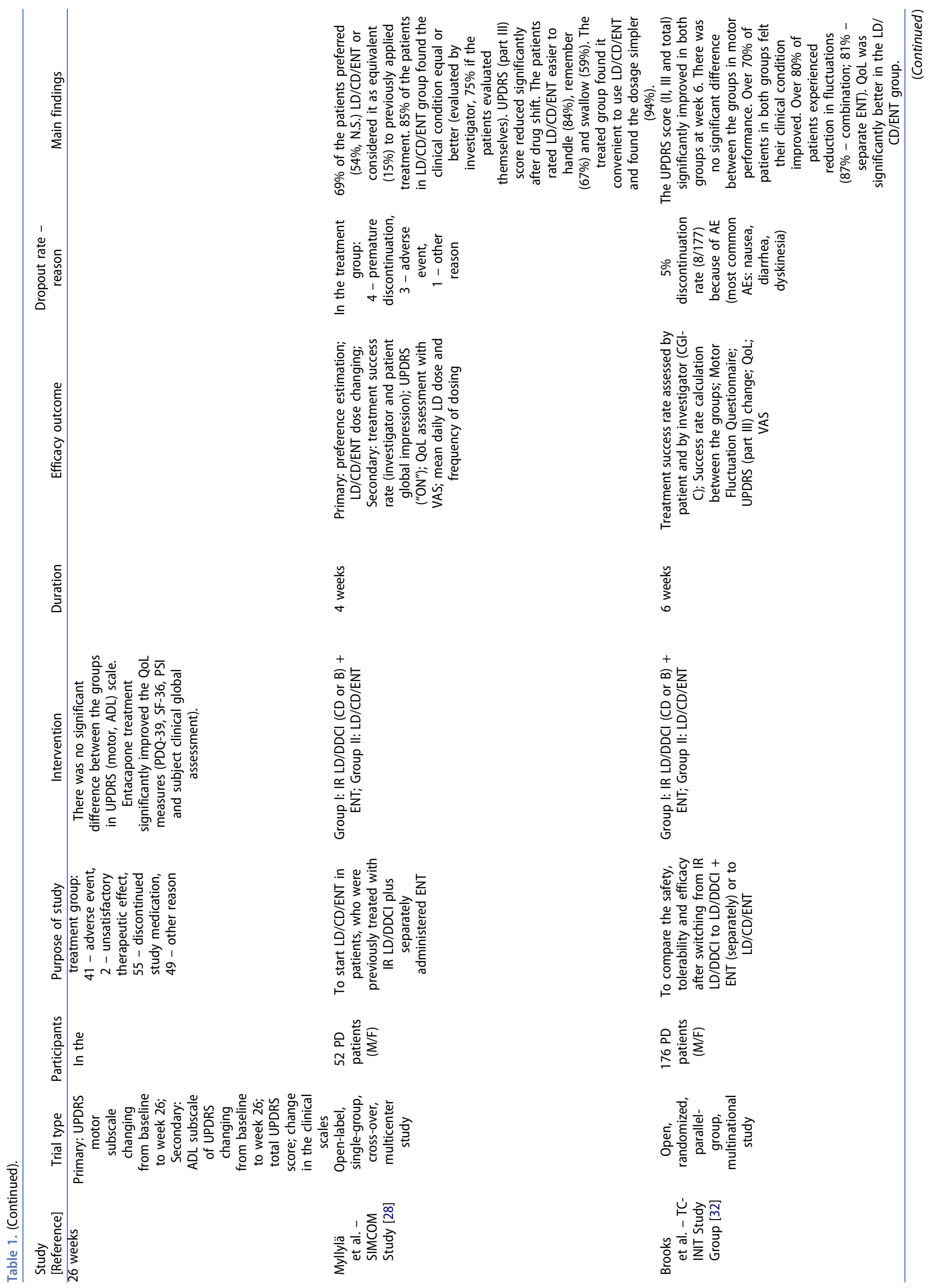




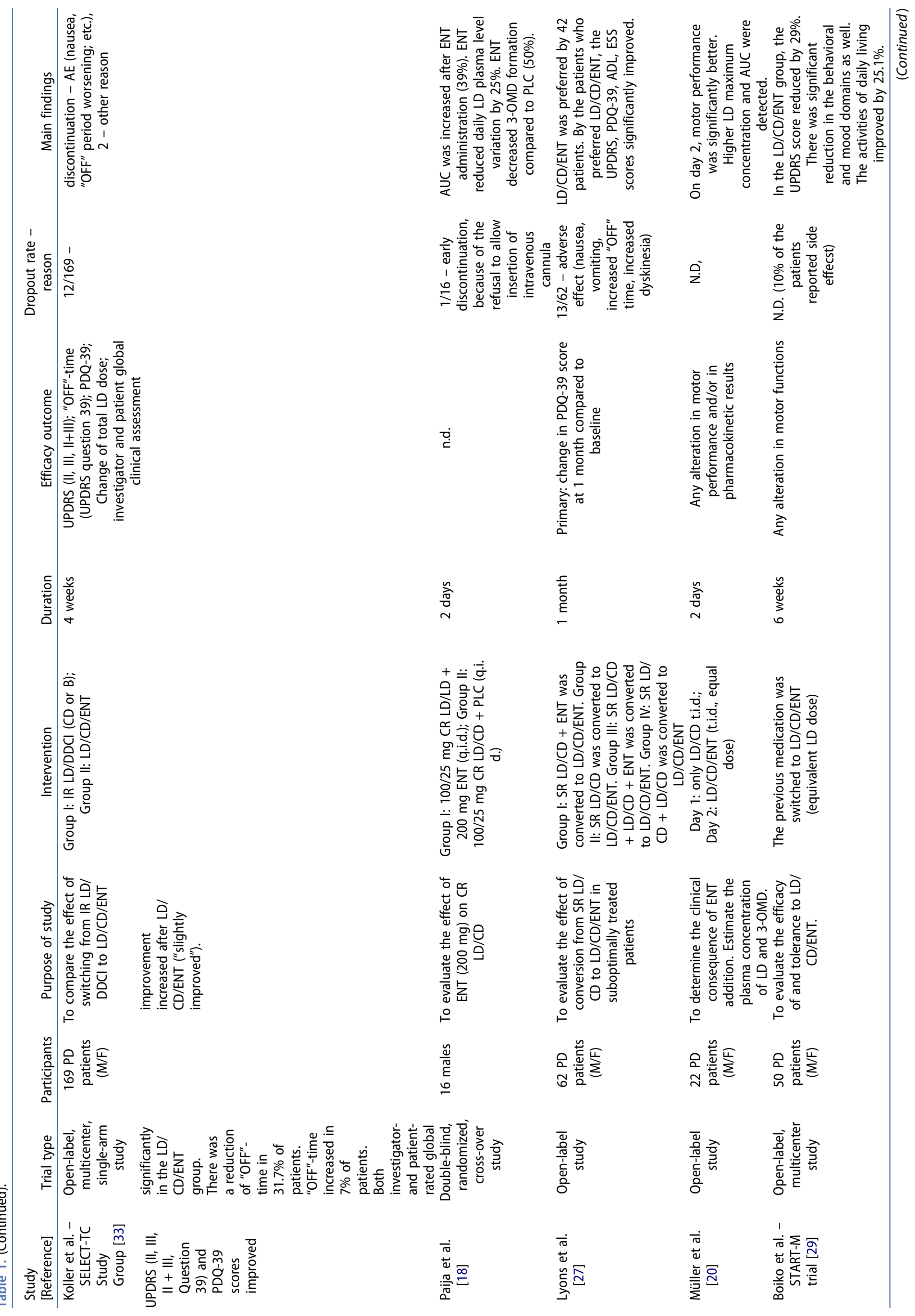




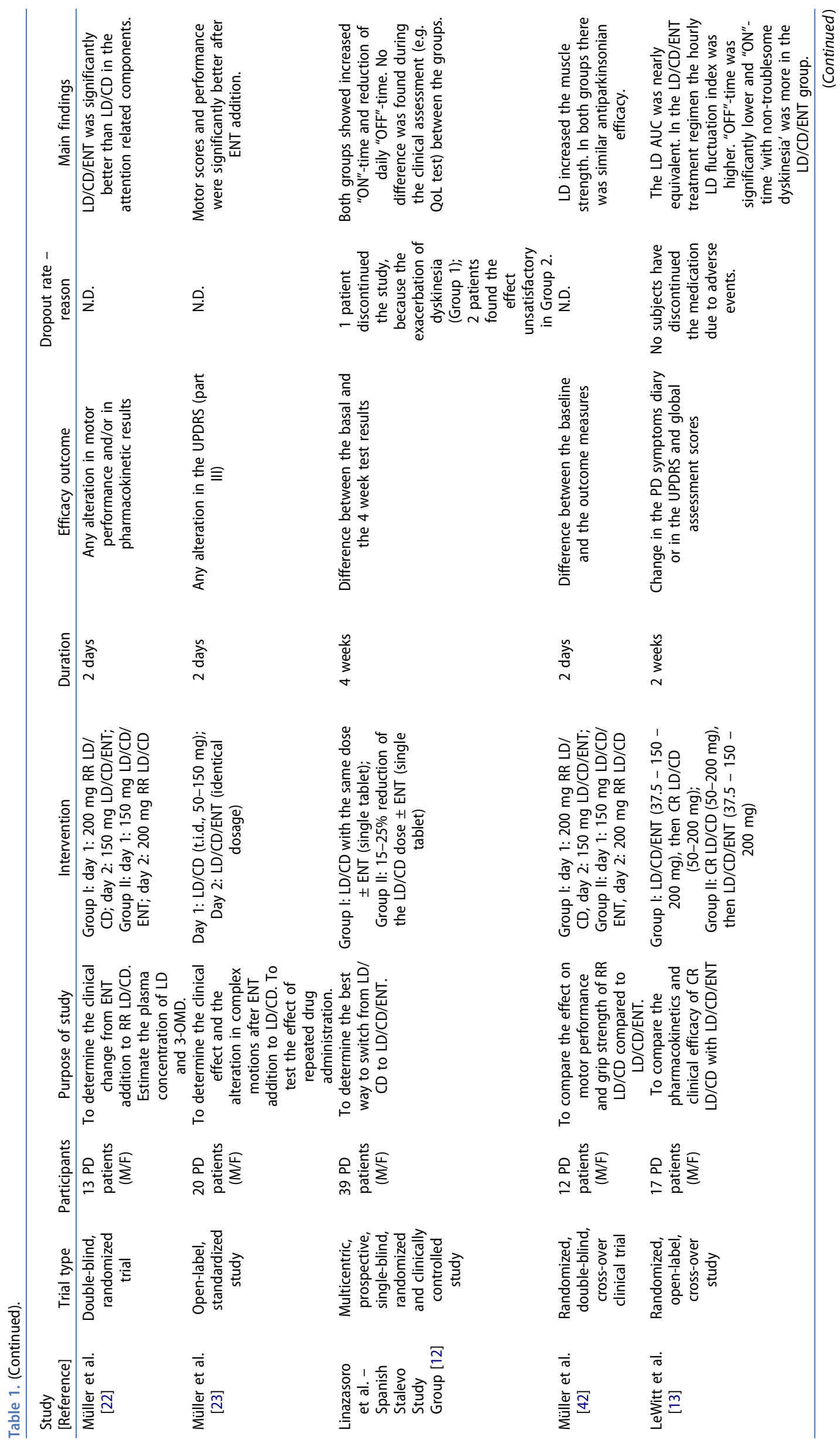




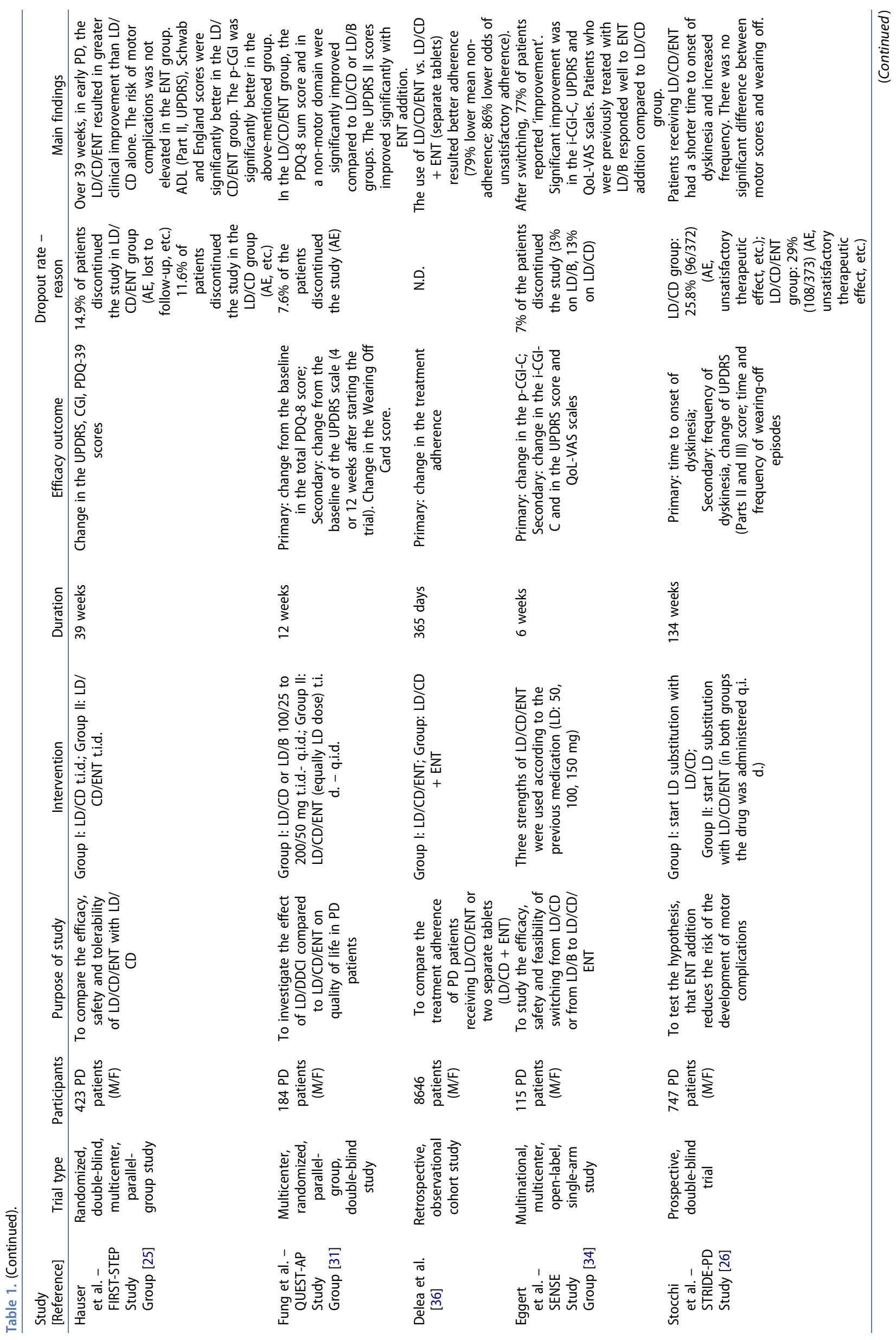




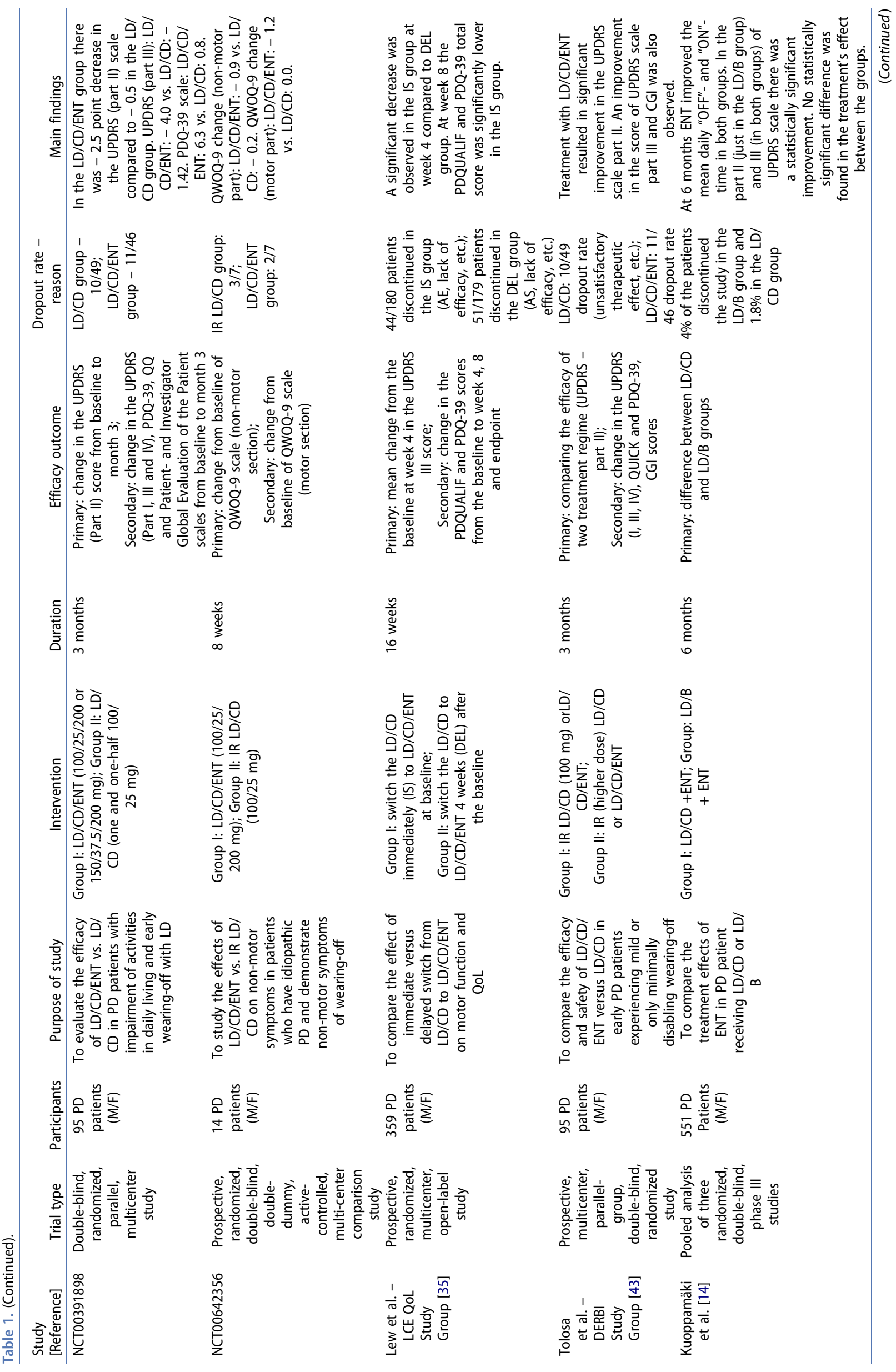




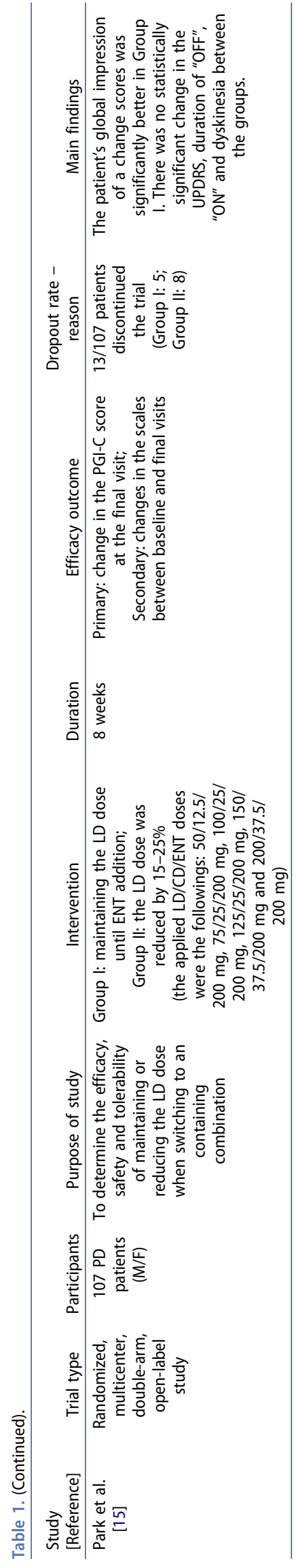

(frequency $-p=0.007$ ) and subject clinical global assessment $(p=0.02)$ [30]. The most affected domains of the PDQ-39 scale were mobility $(p=0.001)$ and ADL $(<0.001)$ [30]. The conversion from $S R L D / C D$ to $L D / C D / E N T$ resulted in a significant improvement in QoL measures after 1 month [27]. The UPDRS (motor score, total score) and the 'mobility', 'ADL', 'emotional', 'cognition' and 'bodily discomfort' domains of PDQ-39 scale improved significantly [27]. These patients also had better scores on the Epworth Sleepiness Scale (ESS) [27]. In the 12 weeks study [31] (184 patients, no or minimal, nondisabling motor fluctuation), the effect of LD/DDCl compared to LD/CD/ ENT on the quality of life was investigated. The applied PDQ-8 scale significantly improved in the LD/CD/ENT group $(p=0.021)$ [31]. The most affected parts of the PDQ-8 scale were 'depression' $(p=0.025)$, 'close personal relationship' $(p=0.037)$, 'communication' ( $p=0.007)$ and 'social stigma' $(p=0.033)$ [31]. In another comparative study [32], over $70 \%$ of the patients in both groups (LD/CD + ENT and. LD/CD/ENT) felt that their clinical condition was better after the switch from the previously applied medication (LD/DDCl). Over $80 \%$ of patients experienced a reduction of fluctuations $(87 \%$ combination; $81 \%$ - separate ENT) [32]. In the SELECT-TC study [33], the effect of switching from IR LD/DDCI to LD/ CD/ENT on QoL was estimated in a 4 week study of patients with wearing-off. The total score of the PDQ-39 scale improved significantly $(p=<0.001)$. Most of the patients reported a slight improvement on the p-CGl scale (34.9\%) [33]. Consistently with these results in a similar study [29], the activities of daily living improved by $25.1 \%$ as well. The effect of the switch from different $D D C l$ inhibitors (LD/CD or $\mathrm{LD} / \mathrm{B})$ to $\mathrm{LD} / \mathrm{CD} / \mathrm{ENT}$ was tested as well [34]. It was found that after switching $77 \%$ of patients reported 'improvement' (p-CGI: LD/CD to LD/CD/ENT: $p=0.008$; LD/B to LD/CD/ENT: $\mathrm{p}=<0.0001)$. There was a significant improvement in the i-CGI-C, UPDRS and QoL-VAS scales as well [34]. Furthermore, it seems that an immediate switch (IS) from LD/CD to LD/CD/ ENT, compared to a delayed switch, has more advantages in terms of QoL [35]. At week 8, the PDQUALIF $(p=0.0133)$ and PDQ-39 $(p=0.0136)$ total scores were significantly lower in the IS group [35].

In the SIMCOM study [28], the effect of the switch from separately administered LD/CD + ENT to LD/CD/ENT (single tablet) was tested. $69 \%$ of the patients preferred $(54 \%$, N.S.) LD/CD/ENT or considered it equivalent (15\%) to previously applied treatments (N.S.). Eighty-five percent of the patients in the LD/CD/ENT group found the clinical condition equal or better (evaluated by investigator, $75 \%$ if the patients evaluated themselves). The patients rated LD/CD/ENT easier to handle $(84 \%)$, remember (67\%) and swallow (59\%) [28]. The treated group found it convenient to use LD/CD/ENT and found the dosage simpler (94\%) [28]. In a previously mentioned comparative study (TC-INIT) [32] the QoL was significantly better as well in the LD/CD/ENT group (CGI-C scale: 'very much improved' - LD/CD + ENT (4\%) versus LD/ CD/ENT (12\%) compared to LD/CD + ENT group [32]. Furthermore, an important large retrospective study [36] was performed which tested the therapeutic adherence between the separate (LD/CD + ENT) and single tablet 


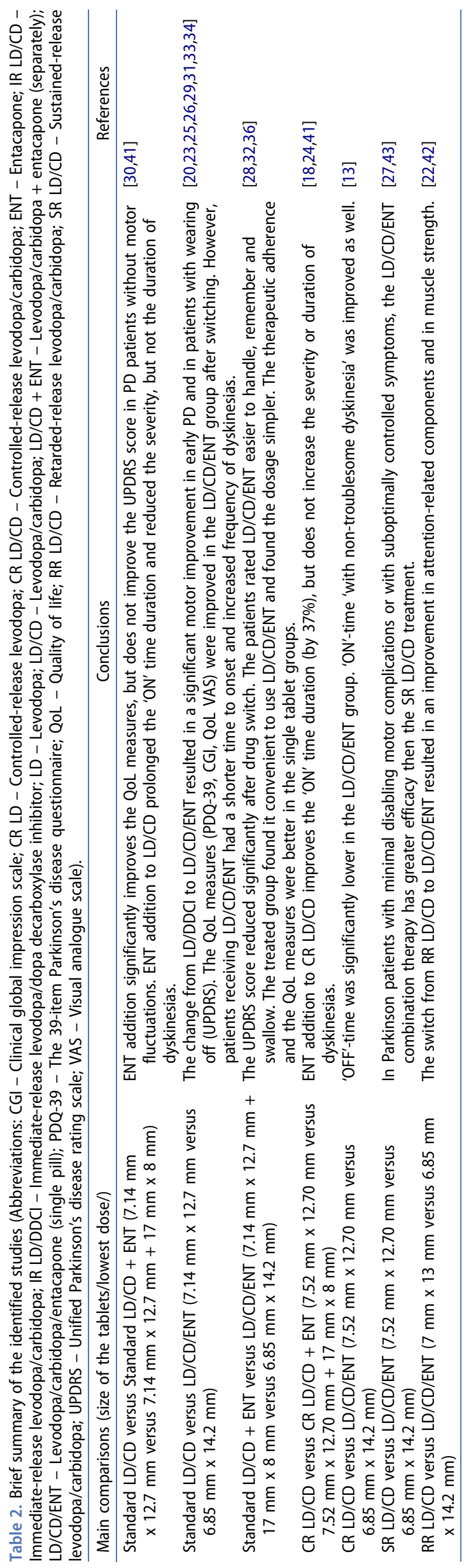


(LD/CD/ENT) forms. The use of LD/CD/ENT vs. LD/CD + ENT (separate tablets) resulted in better adherence $(79 \%$ lower mean non-adherence; $86 \%$ lower odds of unsatisfactory adherence) [36]. As a conclusion, some studies suggest that the single tablet form results in a better QoL; however, no strong evidence is available so far.

\section{Conclusions}

The identified clinical trials provided data on the clinical and pharmacokinetic efficacy of ENT addition. Only a minority of the identified studies $([28,32,36])$ compared the administration mode of ENT. The SIMCOM study proved that $69 \%$ of the patients preferred (54\%, not significant) or considered equivalent (15\%) the LD combination in a single tablet [28]. Additionally, they felt their own clinical condition (85\%) was significantly improved [28]. As opposed to separate dosing, patients found it easier to swallow $(59 \%)$, handle $(84 \%)$ or remember (67\%) [28]. In the TC-INIT study, it was demonstrated that the QoL measures were significantly better in the LD/CD/ENT group compared to the LD/CD + ENT group [32]. Furthermore, the only one involved cohort study (which included 8646 patients) found better patient adherence in the LD/CD/ENT combination group [36]. In conclusion, in PD patients where impaired motor performance justifies the addition of ENT to the previously applied treatment regimen, administration of the single tablet form is suggested.

\section{Expert opinion}

Although many factors (e.g. familial, financial, social) influence the adherence of a PD patient to the applied medication, the importance of non-motor symptoms should be emphasized. The prevalence of depression in Parkinson's disease is 30-40\% [37] and furthermore, around $30-40 \%$ of patients fulfill the diagnostic criteria of cognitive impairment (cumulative prevalence: up to 78\%) [38]. These two non-motor symptoms have been demonstrated to have a relation with drug nonadherence in Parkinson's disease, and therefore optimization and simplification (single pills) of dopaminergic therapy is very important to achieve the optimal therapeutic effects $[39,40]$.

This systematic review summarizes the most important comparative studies regarding ENT administered separately and in combination. During these studies, the administered medicines were closely controlled (no medication error remained unexplained), the UPDRS score did not differ significantly between the LD/CD/ENT versus LD/CD + ENT (separately) groups [32]. Nonetheless, the majority of patients strongly preferred the combination products [28,32]. They felt it easier to handle, swallow, and to remember the appropriate dose [28]. The majority of the performed studies showed a reduction of daily levodopa dose, not only in PD patients who previously were not treated with ENT, but also after switching between separately administered ENT to a combination tablet [18,20-34,36,41-43].

From the pharmacokinetic perspective, there is a need for optimal timing of the oral administration of ENT to achieve the highest bioavailability of LD [16]. The possibility of incorrect administration (e.g. less frequent) of ENT is higher with separate tablets [36]. Furthermore, the widely applied products, which have distinct drug-releasing profiles (e.g. extended-, controlled-release), could make the pharmacokinetics more complex with an additionally increased prevalence of suboptimal LD brain concentration and inappropriate motor symptom control [44]. These facts support the hypothesis that ENT administered in combination yields better bioavailability. ENT addition has a risk of worsening dyskinesia intensity. Dyskinesia was one of the most important factors behind the dropouts (Table 2). The majority of studies showed a discrete reduction in the 'OFF' time; however, we think that 'ON' time is more relevant in judging the efficacy of the ENT treatment.

Examining the cost-effectiveness of combination formulations can be a very important future aspect for the patient's and health insurance's budget. A significantly better quality of life, as documented by clinical studies, is capable of increasing the number of active years and reducing the need for hospital care.

In summary, combination treatments (in particular, ENT combinations in the current work) have been shown to be more effective in terms of quality of life compared to separately administered drugs. During the disease course, cognitive and other non-motor problems, along with motor symptoms, become the leading reasons for non-adherence to medication and not appropriate movement control [36]. The final conclusion of this systematic literature review is that switching to combination ENT treatment in PD patients with end-of-dose wearing-off phenomena is a good option to achieve better QoL. However, patients with motor complications should be reevaluated from time to time regarding instrumental therapies for advanced disease stages [45].

\section{Funding}

This work was supported by the Hungarian Brain Research Program (No. 2017-1.2.1-NKP-2017-00002_VI/4), the Hungarian National Research, Development and Innovation Office (NKFIH) through project GINOP 2.3.2-15-2016-00034 and The Ministry of Human Capacities, Hungary through grant TUDFO/47138-1/2019-ITM.

\section{Declaration of interest}

The authors have no other relevant affiliations or financial involvement with any organization or entity with a financial interest in or financial conflict with the subject matter or materials discussed in the manuscript apart from those disclosed.

\section{Reviewer disclosures}

Peer reviewers on this manuscript have no relevant financial or other relationships to disclose.

\section{References}

Papers of special note have been highlighted as either of interest $(\cdot)$ or of considerable interest $(\cdot \bullet)$ to readers.

1. Beitz JM. Parkinson's disease: a review. Front Biosci (Schol Ed). 2014;6:65-74.

2. Kalia LV, Lang AE. Parkinson's disease. Lancet. 2015;386:896-912. 
- An excellent summary of important aspects of Parkinson's disease (symptoms, pathology, epidemiology, genetics, treatment)

3. Dickson DW. Neuropathology of Parkinson disease. Parkinsonism Relat Disord. 2018;46:S30-S33.

- Neuropathology of Parkinson's disease. The author focuses on the most important neuropathological features of PD based upon personal experience as well. The article also includes cell biological and animal experimental data.

4. Salamon A, Zádori D, Szpisjak L, et al. Neuroprotection in Parkinson's disease: facts and hopes. J Neural Transm (Vienna). 2019. DOI:10.1007/s00702-019-02115-8.

5. Dietrichs $E$, Odin P. Algorithms for the treatment of motor problems in Parkinson's disease. Acta Neurol Scand. 2017;136:378-385.

- Algorithm for the treatment of Parkinson's disease. The authors propose an alternative for optimal treatment of Parkinsonian patients.

6. Marsala SZ, Gioulis M, Ceravolo R, et al. A systematic review of catechol-0-methyltransferase inhibitors: efficacy and safety in clinical practice. Clin Neuropharmacol. 2012;35:185-190.

7. Annus Á, Vécsei L. Spotlight on opicapone as an adjunct to levodopa in Parkinson's disease: design, development and potential place in therapy. Drug Des Devel Ther. 2017;11:143-151.

8. Salamon A, Zádori D, Szpisjak L, et al. Opicapone for the treatment of Parkinson's disease: an update. Expert Opin Pharmacother. 2019;20:2201-2207.

9. Chaudhuri KR, Healy DG, Schapira AH, et al. Non-motor symptoms of Parkinson's disease: diagnosis and management. Lancet Neurol. 2006;5:235-245.

- An excellent summary paper detailing the non-motor symptoms of Parkinson's disease.

10. Palmer CS, Nuijten MJ, Schmier JK, et al. Cost effectiveness of treatment of Parkinson's disease with entacapone in the United States. Pharmacoeconomics. 2002;20:617-628.

11. Liberati A, Altman DG, Tetzlaff J, et al. The PRISMA statement for reporting systematic reviews and meta-analyses of studies that evaluate healthcare interventions: explanation and elaboration. BMJ. 2009;339:b2700.

-. A manuscript detailing the rules for writing a PRISMA review

12. Linazasoro G, Kulisevsky J, Hernández B, et al. Should levodopa dose be reduced when switched to stalevo? Eur J Neurol. 2008;15:257-261.

- From a clinical perpective, the aim of this important study was to determine the best way to switch from LD/CD to LD/CD/ENT. The reduction of the LD dose after switching did not result in the deterioration of motor performance.

13. LeWitt PA, Jennings $D$, Lyons $K E$, et al. Pharmacokineticpharmacodynamic crossover comparison of two levodopa extension strategies. Mov Disord. 2009;24:1319-1324.

14. Kuoppamäki $M$, Leinonen $M$, Poewe W. Efficacy and safety of entacapone in levodopa/carbidopa versus levodopa/benserazide treated Parkinson's disease patients with wearing-off. J Neural Transm (Vienna). 2015;122:1709-1714.

15. Park J, Kim Y, Youn J, et al. Levodopa dose maintenance or reduction in patients with Parkinson's disease transitioning to levodopa/ carbidopa/entacapone. Neurol India. 2017;65:746-751.

- A great study, which tries to determine the efficacy, safety and tolerability of the maintenance or reduction of the LD dose during a switch to an ENT containing combination.

16. Iwaki H, Nishikawa N, Nagai M, et al. Pharmacokinetics of levodopa/ benserazide versus levodopa/carbidopa in healthy subjects and patients with Parkinson's disease. Neurol Clin Neurosci. 2015;3:68-73.
.- An excellent paper about the different pharmacokinetic profiles of $L D$ after LD/CD and LD/B administration in healthy subjects.

17. Heikkinen $H$, Varhe $A$, Laine $T$, et al. Entacapone improves the availability of L-dopa in plasma by decreasing its peripheral metabolism independent of L-dopa/carbidopa dose. Br J Clin Pharmacol. 2002;54:363-371.

.- The purpose of this study was to evaluate the effect of ENT (200 $\mathrm{mg}$ ) in addition to standard LD/CD. They found that ENT increased AUC and decreased 3-OMD formation compared to PLC.

18. Paija O, Laine K, Kultalahti ER, et al. Entacapone increases levodopa exposure and reduces plasma levodopa variability when used with Sinemet CR. Clin Neuropharmacol. 2005;28:115-119.

-. To evaluate the effect of ENT ( $200 \mathrm{mg}$ ) in addition to CR LD/ CD. AUC increased, the 3-OMD level and daily LD plasma level decreased after ENT administration.

19. Kuoppamäki M, Korpela K, Marttila R, et al. Comparison of pharmacokinetic profile of levodopa throughout the day between levodopa/carbidopa/entacapone and levodopa/carbidopa when administered four or five times daily. Eur J Clin Pharmacol. 2009;65:443-455.

.. The purpose of this study was to compare plasma LD concentrations after repeated $L D / C D$ and $L D / C D / E N T$ doses.

20. Müller T, Erdmann $C$, Muhlack $S$, et al. Pharmacokinetic ehavior of levodopa and 3-O-methyldopa after repeat administration of levodopa/carbidopa with and without entacapone in patients with Parkinson's disease. J Neural Transm (Vienna). 2006;113:1441-1448.

21. Müller T, Erdmann C, Muhlack $S$, et al. Inhibition of catechol-O-methyltransferase contributes to more stable levodopa plasma levels. Mov Disord. 2006;21:332-336.

22. Müller T, Ander L, Kolf K, et al. Comparison of $200 \mathrm{mg}$ retarded release levodopa/carbidopa - with $150 \mathrm{mg}$ levodopa/carbidopa/ entacapone application: pharmacokinetics and efficacy in patients with Parkinson's disease. J Neural Transm (Vienna). 2007;114:1457-1462.

- Clinical and pharmacokinetic change assessment after ENT addition to RR LD/CD. In the LD/CD/ENT group members performed significantly better in the attention-related tasks.

23. Müller T, Erdmann C, Muhlack S, et al. Entacapone improves complex movement performance in patients with Parkinson's disease. J Clin Neurosci. 2007;14:424-428.

24. Stocchi F, Barbato L, Nordera G, et al. Entacapone improves the pharmacokinetic and therapeutic response of controlled release levodopa/carbidopa in Parkinson's patients. J Neural Transm (Vienna). 2004;111:173-180.

25. Hauser RA, Panisset M, Abbruzzese G, et al. Double-blind trial of levodopa/carbidopa/entacapone versus levodopa/carbidopa in early Parkinson's disease. Mov Disord. 2009;24:541-550.

- A well-written paper, which concluded that over 39 weeks, in early PD patients, the LD/CD/ENT combination could result in a greater clinical improvement, without the augmentation of motor complication development.

26. Stocchi F, Rascol O, Kieburtz K, et al. Initiating levodopa/carbidopa therapy with and without entacapone in early Parkinson disease: the STRIDE-PD study. Ann Neurol. 2010;68:18-27.

.. This trial tested the hypothesis that ENT addition could reduce the risk of developing motor complications. Contrarily, they found that the LD/CD/ENT combination resulted in a shorter time to the onset of dyskinesia and increased the frequency.

27. Lyons KE, Pahwa R. Conversion from sustained release carbidopa/ levodopa to carbidopa/levodopa/entacapone (stalevo) in Parkinson disease patients. Clin Neuropharmacol. 2006;29:73-76. 
.. Conversion from SR LD/CD to LD/CD/ENT. For the majority of patients, quality of life and motor performance improved after shifting.

28. Myllylä V, Haapaniemi T, Kaakkola S, et al. Patient satisfaction with switching to Stalevo: an open-label evaluation in PD patients experiencing wearing-off (Simcom Study). Acta Neurol Scand. 2006;114:181-186.

- The purpose of this study was to start LD/CD/ENT in patients who were previously treated with IR LD/DDCI plus separately administered ENT. $69 \%$ of the patients preferred (54\%, N.S.) LD/CD/ENT or considered it as equivalent (15\%) to previously applied treatment (N.S.).

29. Boiko AN, Batysheva TT, Minaeva NG, et al. Use of the new levodopa agent Stalevo (levodopa/carbidopa/entacapone) in the treatment of Parkinson's disease in out-patient clinical practice (the START-M open trial). Neurosci Behav Physiol. 2008;38:933-936.

30. Olanow CW, Kieburtz K, Stern M, et al. Double-blind, placebo-controlled study of entacapone in levodopa-treated patients with stable Parkinson disease. Arch Neurol. 2004;61:1563-1568.

-. The clinical effect of ENT addition to LD in PD patients without motor complications was tested. They found that ENT addition significantly improved the QoL measures, however, the UPDRS score remained relatively unchanged.

31. Fung VS, Herawati L, Wan Y, et al. Quality of life in early Parkinson's disease treated with levodopa/carbidopa/entacapone. Mov Disord. 2009:24:25-31.

- Comparison of LD/DDCI with LD/CD/ENT on quality of life in PD patients. There was a significant improvement in the PDQ-8 sum score and in a non-motor domain in the LD/CD/ENT group.

32. Brooks DJ, Agid Y, Eggert K, et al. Treatment of end-of-dose wearing-off in parkinson's disease: stalevo (levodopa/carbidopa/entacapone) and levodopa/DDCl given in combination with Comtess/ Comtan (entacapone) provide equivalent improvements in symptom control superior to that of traditional levodopa/DDCl treatment. Eur Neurol. 2005;53:197-202.

- Switching from IR LD/DDCI to LD/DDCI + ENT (separately) or to LD/CD/ENT. There was no significant difference between the groups in the motor performance. However, the majority of patients preferred the LD/CD/ENT single tablet form.

33. Koller W, Guarnieri M, Hubble J, et al. An open-label evaluation of the tolerability and safety of Stalevo (carbidopa, levodopa and entacapone) in Parkinson's disease patients experiencing wearingoff. J Neural Transm (Vienna). 2005;112:221-230.

34. Eggert K, Skogar O, Amar K, et al. Direct switch from levodopa/ benserazide or levodopa/carbidopa to levodopa/carbidopa/entacapone in Parkinson's disease patients with wearing-off: efficacy, safety and feasibility-an open-label, 6-week study. J Neural Transm (Vienna). 2010;117:333-342.

35. Lew MF, Somogyi M, McCague K, et al. Immediate versus delayed switch from levodopa/carbidopa to levodopa/carbidopa/entacapone: effects on motor function and quality of life in patients with Parkinson's disease with end-of-dose wearing off. Int J Neurosci. 2011;121:605-613.

.- The purpose of this study was to compare the effect of an immediate versus delayed switch from LD/CD to LD/CD/ENT on motor function and QoL. The immediate switch resulted in an earlier decrease in the UPDRS score and led to better QoL scores.
36. Delea TE, Thomas SK, Hagiwara M, et al. Adherence with levodopa/ carbidopa/entacapone versus levodopa/carbidopa and entacapone as separate tablets in patients with Parkinson's disease. Curr Med Res Opin. 2010;26:1543-1552.

- Retrospective, observational cohort study which collected data on the adherence of PD patients receiving LD/CD/ENT or two separate tablets (LD/CD + ENT). The single tablet form led to better therapeutic adherence.

37. Aarsland D, Marsh L, Schrag A. Neuropsychiatric symptoms in Parkinson's disease. Mov Disord. 2009;24:2175-2186.

38. Goetz CG, Emre M, Dubois B. Parkinson's disease dementia: definitions, guidelines, and research perspectives in diagnosis. Ann Neurol. 2008;64:(S.2):81-92.

-. A great article which focuses on the important aspects of dementia related to Parkinson's disease.

39. Insel K, Morrow D, Brewer B, et al. Executive function, working memory, and medication adherence among older adults. J Gerontol B Psychol Sci Soc Sci. 2006;61:102-107.

40. Stoehr GP, Lu SY, Lavery L, et al. Factors associated with adherence to medication regimens in older primary care patients: the Steel Valley Seniors Survey. Am J Geriatr Pharmacother. 2008;6:255-263.

41. Piccini P, Brooks DJ, Korpela K, et al. The catecholO-methyltransferase (COMT) inhibitor entacapone enhances the pharmacokinetic and clinical response to sinemet $C R$ in Parkinson's disease. J Neurol Neurosurg Psychiatry. 2000;68:589-594.

-. The purpose of this study was to test the clinical consequence of ENT addition to standard LD versus CR LD. UPDRS improvement was higher in the CR LD/CD + ENT group compared to CR LD/CD + PLC group.

42. Müller T, Kolf K, Ander L, et al. Catechol-O-methyltransferase inhibition improves levodopa-associated strength increase in patients with Parkinson disease. Clin Neuropharmacol. 2008;31:134-140.

43. Tolosa E, Hernández B, Linazasoro $G$, et al. Efficacy of levodopa/ carbidopa/entacapone versus levodopa/carbidopa in patients with early Parkinson's disease experiencing mild wearing-off: a randomised, double-blind trial. J Neural Transm (Vienna). 2014;121:357-366.

.. $L D / C D / E N T$ versus $L D / C D$ in early PD patients experiencing mild or only minimally disabling wearing-off. Treatment with LD/CD/ENT resulted in significant improvement in the UPDRS scale part II.

44. Hsu A, Yao HM, Gupta S, et al. Comparison of the pharmacokinetics of an oral extended-release capsule formulation of carbidopalevodopa (IPX066) with immediate-release carbidopa-levodopa $\left(\right.$ Sinemet $\left({ }^{(}\right)$), sustained-release carbidopa-levodopa (Sinemet $\left.{ }^{\circledast}\right)$ $\mathrm{CR})$, and carbidopa-levodopa-entacapone (Stalevo $\left.\left({ }^{(}\right)\right)$. J Clin Pharmacol. 2015;55:995-1003.

45. Antonini A, Stoessl AJ, Kleinman LS, et al. Developing consensus among movement disorder specialists on clinical indicators for identification and management of advanced Parkinson's disease: a multi-country Delphi-panel approach. Curr Med Res Opin. 2018;34:2063-2073.

.. An excellent consensus paper, which emphasizes the importance of early recognition of patients with advanced stage disease to improve the QoL by applying device-aided therapies. 